\title{
Prevalence of CYP2C9 and VKORC1 alleles in the Argentine population and implications for prescribing dosages of anticoagulants
}

\author{
P. Scibona, M.A. Redal, L.G. Garfi, J. Arbelbide, P.F. Argibay and \\ W.H. Belloso \\ Clinical Pharmacology Section, Hematology Section, \\ Internal Medicine Service. \\ Molecular and Genomic Medicine Unit, \\ Basic Sciences and Experimental Medicine. \\ Hospital Italiano of Buenos Aires, Buenos Aires, Argentina \\ Corresponding author: P. Scibona \\ E-mail: paula.scibona@hospitalitaliano.org.ar
}

Genet. Mol. Res. 11 (1): 70-76 (2012)

Received April 4, 2011

Accepted October 5, 2011

Published January 9, 2012

DOI http://dx.doi.org/10.4238/2012.January.9.8

\begin{abstract}
Dicumarinic oral anticoagulants have a narrow therapeutic range and a great individual variability in response, which makes calculation of the correct dose difficult and critical. Genetic factors involved in this variability include polymorphisms of genes that encode the metabolic enzyme CYP2C9 and the target enzyme vitamin $\mathrm{K}$ epoxide reductase complex 1 (VKORC1); these polymorphisms can be associated with reduced enzymatic expression. We examined the frequency of the most relevant variants encoding CYP2C9 (alleles *1, *2 and *3) and VKORC1 (SNP -1639A $>$ G) in the Argentinian population. Molecular typing was performed by PCRRFLP on a randomly selected sample of 101 healthy volunteers from the Hospital Italiano de Buenos Aires gene bank. Fifty-seven subjects were identified as homozygous for CYP2C $9 * 1$ and 14 for $* 2$, while 24 and 5 were heterozygous for $* 2$ and $* 3$ alleles; one individual was a composite heterozygote $(* 2 / * 3)$. When we examined VKORC1, 21 subjects were AA homozygous, 60 were AG heterozygotes and 20 were
\end{abstract}


GG homozygotes. This is the first analysis of genotypic frequencies for CYP2C9 and VKORC1 performed in an Argentinian population. These allele prevalences are similar to what is known for Caucasian population, reflecting the European ancestor of our patient population, coming mostly from Buenos Aires city and surroundings. Knowledge of this prevalence information is instrumental for cost-effective pharmacogenomic testing in patients undergoing oral anticoagulation treatment.

Key words: Anticoagulant; Pharmacogenomics; CYP2C9; VKORC1; Allelic frequency; Argentina

\section{INTRODUCTION}

Coumarin oral anticoagulants (OAC) are widely used drugs for the treatment of venous thrombosis, atrial fibrillation and myocardial infarction and prevention of cardioembolic stroke, among other indications. One of their distinctive pharmacological features is a narrow therapeutic range.

Their efficacy and safety is determined and monitored by the INR (International Normalized Ratio), whose maintenance within desirable values is crucial for therapeutic effectiveness.

Poor anticoagulant control may result in an increase in morbimortality associated with either appearance or recurrence of a thrombotic event or hemorrhage. Up to $50 \%$ of patients under anticoagulation therapy fail in the achievement or maintenance of an adequate INR with administered doses (Hylek et al., 2000). The main reason for this is the great variability that exists in the response to coumarin agents.

There are a number of variables associated with this variability in response. Among them are age, vitamin $\mathrm{K}$ content in the diet, pharmacological interactions, fever, cardiac failure, and hepatocellular damage. In addition, genetic factors that contribute to the explanation of the variability in dose requirements have also been described.

Isozyme CYP2C9 from the cytochrome $\mathrm{P} 450$ complex is a hepatic enzyme that participates in the metabolism of coumarin anticoagulants and many other drugs. The gene is located on chromosome 10q24.2 (GenBanK id1559). The polymorphism of CYP2C9 has been described and there are two frequent allelic variants associated with a decreased enzymatic activity: allele $* 2$, showing a substitution of arginine for cysteine at position 144 in exon 3 (rs1799853), and allele *3, showing a substitution of isoleucine for leucine at position 359 in exon 7 (rs1057910). These variants determine an enzyme with 12 and 5\% activity, respectively, in comparison to the wild-type (CYP2C $9 * 1)$. Both variants have been related to more sensitivity to OAC and thus lower dose requirements (Table 1). Genotype frequencies significantly differ according to the population studied, although in Caucasians, about $1 \%$ are homozygous for the CYP2C $9 * 2$ allele and $22 \%$ are heterozygotes (CYP2C9*1/*2), whereas for the CYP2C $9 * 3$ allele, approximately $0.4 \%$ are homozygotes and $15 \%$ heterozygote (CYP2C $9 * 1 / * 3)$, and approximately $1.4 \%$ of the population has been described as being composed of heterozygotes (CYP2C9*2/*3) (Kamali and Pirmohamed, 2006; Schwarz et al., 2008; Martin, 2009). 
Table 1. CYP2C9 and VKORC1 polymorphisms and sensitivity to a coumarin oral anticoagulant.

\begin{tabular}{lcl}
\hline CYP2C9 & VKORC1 $-1639 \mathrm{G}>\mathrm{A}$ & Sensitivity to warfarin \\
\hline CYP2C $9 * 1$ & GG & Low \\
CYP2C 92 & AG & Intermediate \\
CYP2C9*3 & AA & High \\
\hline
\end{tabular}

On the other hand, the subunit 1 of vitamin $\mathrm{K}$ epoxide reductase (VKORC1) is responsible for the transformation of vitamin K 2,3 epoxide to its reduced form, required for the gamma-carboxylation of the vitamin K-dependent coagulation factors (factors II, VII, IX, and $\mathrm{X})$. Coumarin OACs inhibit the VKORC1 enzyme complex, preventing the regeneration of the reduced form of vitamin $\mathrm{K}$, and exerting in this form its anticoagulant action.

Single nucleotide polymorphisms (SNPs) have been described for the gene (GenBank id79001) encoding VKORC1, many of which are in linkage disequilibrium, related to a decrease in enzymatic expression and thus resulting in a reduction in the OAC dose required. The VKORC1 complex gene is located on chromosome 16p12-21q, where one of the most relevant variants is at position -1639 of the promoter region, with a $\mathrm{G}>\mathrm{A}$ substitution (rs9923231) (D’Andrea et al., 2005; Yuan et al., 2005) (Table 1).

The allelic frequencies of the VKORC1 complex vary in different populations. Regarding this variant, the reported frequencies for Caucasian populations have been $14 \% \mathrm{AA}$, $47 \% \mathrm{AG}$ and $39 \% \mathrm{GG}$.

Currently, the FDA recommends the performance of a pharmacogenomic test for CYP2C9 variants for every patient about to initiate OAC therapy. The cost-effectiveness of the implementation of this measure requires frequency studies in local populations.

Although, most of Argentinian habitants are of Caucasian descent, to date, there are no formal data about CYP2C9 and VKORC1 allelic frequencies in our country.

The objective of the present study was to determine the frequency of the most relevant variants of the genes encoding cytochrome P450 2C9 (alleles *1, *2 and *3) and the VKORC1 complex (SNP -1639A $>$ G) in the Argentinian population aiming to estimate the potential impact of these polymorphisms in patients treated with coumarin OAC.

\section{MATERIAL AND METHODS}

\section{Study population}

One hundred and one random samples from healthy controls obtained from the Hospital Italiano de Buenos Aires DNA Bank were analyzed.

\section{Sample collection and DNA extraction}

Following an informed consent process, $10 \mathrm{~mL}$ peripheral blood was collected from each subject in 5-mL EDTA tubes. Whole blood samples were stored at $4^{\circ} \mathrm{C}$ until the time of processing. Genomic DNA was extracted and purified using the QIAmp DNA Blood Mini kit (QIAGEN). 


\section{Genotyping of CYP2C9 and VKORC1}

Genotyping of the CYP2C9 and VKORC alleles was performed by PCR-RFLP, according to the techniques described by Ku Chee Sehg et al. (2003) and Wen et al. (2008), respectively, with some modifications. In each reaction, genomic DNA was amplified using the same sequence-specific primers for exons 3 and 7 of the CYP2C9 gene generating 375- and 105-bp amplicons, respectively, and for VKORC1 $-1639 \mathrm{~A}>\mathrm{G}$ polymorphism as described by $\mathrm{Ku}$ Chee Sehg et al. (2003) and Wen et al. (2008), respectively.

Each PCR was performed in a $20-\mu \mathrm{L}$ mixture containing $1 \mathrm{X}$ PCR buffer (Promega), $200 \mathrm{mM}$ dNTPs (Fermentas), $0.2 \mu \mathrm{M}$ of each primer (Eurofins MWG Operon), $1 \mathrm{U}$ Taq polymerase (Promega), and 100 ng genomic DNA. Reactions were performed in an MJ Research Thermocycler (PTC 100). Cycling parameters for CYP2C9 PCR were as previously published (Ku Chee Sehg et al., 2003). Cycling parameters for VKORC were as follows: initial denaturation at $94^{\circ} \mathrm{C}$ for $4 \mathrm{~min}$ and 30 cycles of $94^{\circ} \mathrm{C}$ for $45 \mathrm{~s}, 56^{\circ} \mathrm{C}$ for $30 \mathrm{~s}$, and $72^{\circ} \mathrm{C}$ for $45 \mathrm{~s}$, and a final extension step at $72^{\circ} \mathrm{C}$ for $10 \mathrm{~min}$.

Aliquots of PCR products were digested with restriction enzymes (Fermentas), AvaII for CYP2C9*2, KpnI for CYP2C9*3 and $M s p I$ for VKORC1, at $37^{\circ} \mathrm{C}$ overnight to ensure complete digestion. The DNA fragments were electrophoresed on a $3 \%$ agarose gel stained with ethidium bromide, and bands were visualized with a UV transilluminator (Table 2).

\section{Table 2. CYP2C9 genotyping with restriction enzymes.}

\begin{tabular}{|c|c|c|c|c|c|c|c|c|c|c|c|}
\hline \multicolumn{12}{|c|}{ Genotypes } \\
\hline \multicolumn{2}{|c|}{$* 1 / * 1$} & \multicolumn{2}{|c|}{$* 1 / * 2$} & \multicolumn{2}{|c|}{$* 1 / * 3$} & \multicolumn{2}{|c|}{$* 2 / * 2$} & \multicolumn{2}{|c|}{$* 2 / * 3$} & \multicolumn{2}{|c|}{$* 3 / * 3$} \\
\hline Ava & $K p n$ & Ava & $K p n$ & Ava & Kpn & Ava & $K p n$ & Ava & $K p n$ & Ava & Kpn \\
\hline & 105 & 375 & 105 & & 105 & 375 & 105 & 375 & 105 & & \\
\hline 296 & & 296 & & 296 & 85 & & & 296 & 85 & 296 & 85 \\
\hline 79 & & 79 & & 79 & 20 & & & 79 & 20 & 79 & 20 \\
\hline
\end{tabular}

In the 375-bp amplicon of 2C9, cleavage with the AvaII enzyme in the presence of alleles $* 1$ and $* 3$ generated two fragments of 296 and $79 \mathrm{bp}$, while allele $* 2$ showed the 375 -bp amplicon intact. The 105-bp sequence, after digestion with KpnI, generated two fragments of 85 and $20 \mathrm{bp}$ in the presence of the CYP2C $9 * 3$ allele, while in the presence of CYP2C $9 * 1$ and *2 alleles, the 105-bp fragment remained unchanged (Table 2).

For the detection of the $-1639 \mathrm{G}$ allele of the VKORC1 sequence, specific primers were used, generating a 290-bp amplicon that after digestion with the $M s p I$ enzyme resulted in two fragments of 123 and $167 \mathrm{bp}$, while in the presence of the A allele, the original 290-bp sequence remained unchanged since it is resistant to cleavage. The results were verified through bidirectional direct sequencing of the PCR products.

\section{Statistical analysis}

Allelic and genotypic frequencies were expressed in absolute and percentage values in the study population. The results obtained were further evaluated for Hardy-Weinberg equilibrium in accordance with the expected frequencies for a Caucasian population, based on previously published results. 


\section{RESULTS}

All samples were genotyped according to the method previously described. Among the 101 subjects analyzed, $53.4 \%$ (54) were females and $47.6 \%$ (47) were males with a median age of 35 and a range of 21-67 years.

In our population, $56 \%(57)$ showed the wild-type CYP2C $9 * 1$, while $23 \%(24)$ and $4 \%$ (5) were heterozygous for $* 2$ and $* 3$ alleles, respectively. In this population, $13 \%$ (14) were homozygous for the $* 2$ allele, whereas none homozygous for the $* 3$ allele was detected, and less than $1 \%$ of the population were composite heterozygotes $(* 2 / * 3)$ (Figure 1). Regarding VKORC1, a frequency of $20 \%$ (21) was found for AA homozygotes, $60 \%$ (60) for AG heterozygotes and 19\% (20) for GG homozygotes (Figure 2).

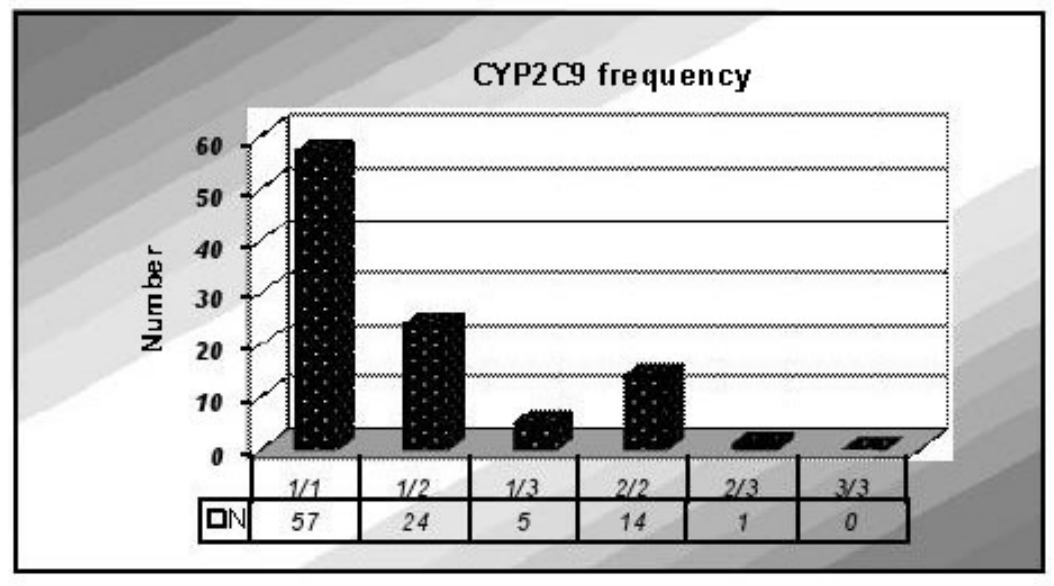

Figure 1. Genotype frequencies of CYP2C9 in the population studied.

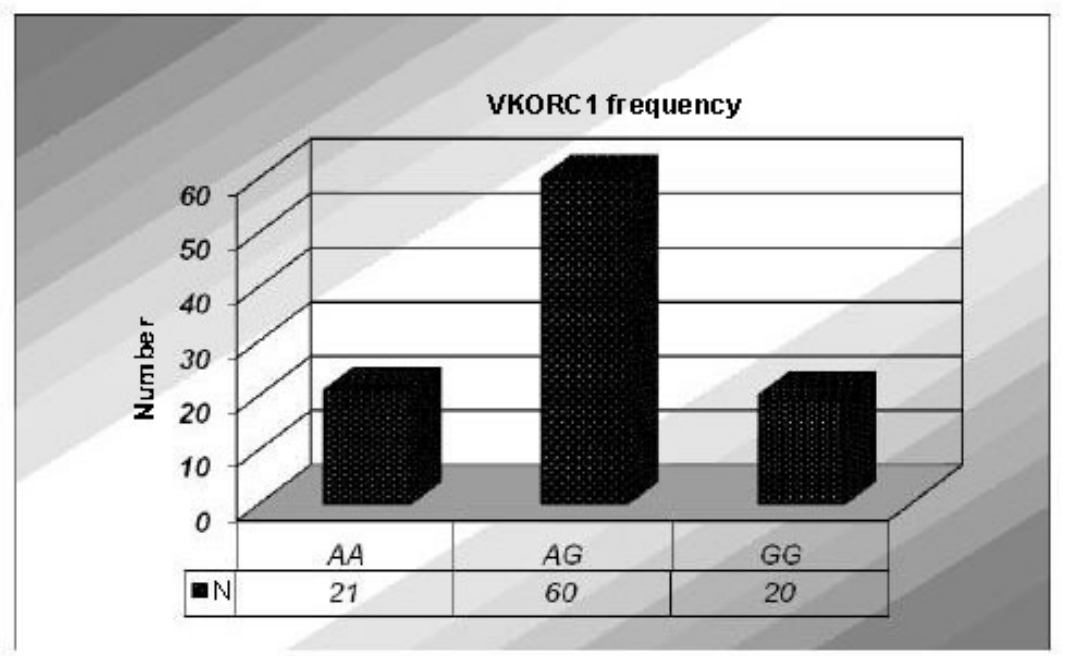

Figure 2. Genotype frequencies of VKORC1 in the population studied. 
Combining the frequencies of the CYP2C9 and VKORC polymorphisms, we found that among the VKORC1 AA allele carriers, $62 \%$ were homozygous for the CYP2C9*1 allele, $24 \%$ were $* 1 / * 2$ heterozygotes and $14 \%$ were homozygous for the $* 2$ allele.

Among the VKORC1 AG heterozygous individuals, 58\% were CYP2C9*1 homozygotes, $22 \%$ were $* 1 / * 2$ heterozygotes, $10 \%$ were homozygous for the $* 2$ allele, $8 \%$ were $* 1 / * 3$ heterozygotes and less than $2 \%$ were $* 2 / * 3$ heterozygotes.

Among the VKORC1 GG homozygotes, $45 \%$ were CYP2C9*1 homozygotes, $30 \%$ were $* 1 / * 2$ heterozygotes, and $25 \%$ were homozygous for the $* 2$ variant.

\section{DISCUSSION}

A significant proportion of interindividual variability in the response to coumarin anticoagulant drugs has been attributed to the presence of polymorphisms in CYP2C9 and VKORC1 genes, although the overall impact varies between different populations in relation to differential frequencies of those genetic variants.

Thus, it has become undebatably important to perform frequency studies of potentially relevant polymorphisms in different populations in order to establish the relative importance of specific allelic variants and to estimate the usefulness of genetic analysis in a particular scenario. On the other hand, the absence or minimal penetrance of particular alleles may preclude their further analysis due to cost-effectiveness considerations, as is the case for the $* 2$ variant of CYP2C9 in the Oriental population.

In the present study, we analyzed the frequency of the allelic variants for both CYP2C9 and VKORC1 in the Argentinian population, information that, to the best of our knowledge, has not been reported before.

We found that the genotypic frequencies for CYP2C9 and VKORC1 were similar to those described for other populations of Caucasian origin. The significant presence of alleles associated with an increased susceptibility to coumarin agents may warrant the use of pharmacogenomic analysis in the Argentinian population ahead of the initiation of OAC therapy, as has been recommended elsewhere by regulatory agencies.

A limitation of our findings is that most of the samples analyzed come from a particular region (Buenos Aires city and its surroundings), which may impede complete extrapolation to the entire Argentinian population, although genetic origin may be similar for most of the major cities in our country.

Even when pharmacogenomic analysis has been formally recommended for patients undergoing warfarin therapy, currently available data for other coumarin drugs such as acenocumarol are more limited, although this is important since acenocumarol is the most frequently used OAC in our country. Nevertheless, acenocumarol pharmacokinetic and pharmacodynamic characteristics are similar to those of warfarin, and as such the information regarding the allele frequencies may become similarly relevant.

Pharmacogenomic information may help in the prediction of over-anticoagulation and an excess risk of bleeding for patients harboring the variants of CYP2C9 and VKORC1 associated with a decreased expression of the corresponding enzyme, although clear recommendations regarding the management of these patients are not widely available. In the meantime, whether to reduce the initial dose or initiate an alternative drug, relies mostly on clinical decisions (Taube et al., 2000). Notwithstanding, a recent publication has shown a significant 
reduction of clinical outcomes (all-cause and disease-specific hospitalization rates) when the initial dose of warfarin was guided by pharmacogenomic testing. Further confirmation of these results in different settings and with other coumarin agents would be of utmost help in the applicability of genetic data in day-to-day anticoagulation treatment (Epstein et al., 2010).

In summary, we demonstrated that the frequencies of CYP2C9 and VKORC1 alleles found in an Argentinian population are similar to those reported in other populations of Caucasian origin, thus providing support for further evaluation of the practical consequences of pharmacogenomic evaluation in patients undergoing oral anticoagulant therapy in our setting.

\section{Conflict of interest}

Authors declare no competing interests, either financial or non-financial. No financial support was received for the preparation of this manuscript.

\section{REFERENCES}

D'Andrea G, D'Ambrosio RL, Di Perna P, Chetta M, et al. (2005). A polymorphism in the VKORC1 gene is associated with an interindividual variability in the dose-anticoagulant effect of warfarin. Blood 105: 645-649.

Epstein RS, Moyer TP, Aubert RE, O'Kane DJ, et al. (2010). Warfarin genotyping reduces hospitalization rates results from the MM-WES (Medco-Mayo Warfarin Effectiveness study). J. Am. Coll. Cardiol. 55: 2804-2812.

Hylek EM, Chang YC, Skates SJ, Hughes RA, et al. (2000). Prospective study of the outcomes of ambulatory patients with excessive warfarin anticoagulation. Arch. Intern. Med. 160: 1612-1617.

Kamali F and Pirmohamed M (2006). The future prospects of pharmacogenetics in oral anticoagulation therapy. Br. $J$. Clin. Pharmacol. 61: 746-751.

Ku Chee Sehg, Gan Gin Gin, J Vijaya Sangkar and Maude Elvira Phipps (2003). Frequency of cytochrome P450 2 C9 (CYP2C9) alleles in three ethnic groups in malaysia. Asian Pac. J. Mol. Biol. 11: 83-91.

Martin JH (2009). Pharmacogenetics of warfarin- is testing clinically indicated? Australian Prescriber 32: 76-80.

Schwarz UI, Ritchie MD, Bradford Y, Li C, et al. (2008). Genetic determinants of response to warfarin during initial anticoagulation. N. Engl. J. Med. 358: 999-1008.

Taube J, Halsall D and Baglin T (2000). Influence of cytochrome P-450 CYP2C9 polymorphisms on warfarin sensitivity and risk of over-anticoagulation in patients on long-term treatment. Blood 96: 1816-1819.

Wen MS, Lee M, Chen JJ, Chuang HP, et al. (2008). Prospective study of warfarin dosage requirements based on CYP2C9 and VKORC1 genotypes. Clin. Pharmacol. Ther. 84: 83-89.

Yuan HY, Chen JJ, Lee MT, Wung JC, et al. (2005). A novel functional VKORC1 promoter polymorphism is associated with inter-individual and inter-ethnic differences in warfarin sensitivity. Hum. Mol. Genet. 14: 1745-1751. 